\title{
GANGRENE ASSOCIATED WITH RHEUMATOID VASCULITIS: A CASE REPORT
}

Vinícius Shinoda Pereira ${ }^{1, \star}$, Mariana Sandy Mada², Samuel dos Santos Bueno ${ }^{2}$, Lesley Ane Roks de Lima1', Juliane Aline Paupitz ${ }^{2}$

1.Centro Universitário de Maringá, Maringá (PR), Brazil. 2.Universidade Federal de Santa Catarina, Florianópolis (SC), Brazil.

${ }^{\star}$ Corresponding author: shinoda.vinicius.pereira@gmail.com

\section{BACKGROUND}

Rheumatoid arthritis (RA) is a chronic inflammatory disease predominant in females between 40 and 60 years. Commonly causes inflammation of small joints of hands and feet. Rheumatoid vasculitis affects about $1 \%$ of patients, usually those with long-standing RA and positive RF. It affects small and medium vessels and may be associated with peripheral neuropathy and digital gangrene. The treatment includes antibiotic therapy and amputation in more severe cases.

\section{CASE REPORT}

White, female, 75 years old, complains of fetid odor in left $(L)$ toes. Was diagnosed more than 10 years ago with rheumatoid arthritis (RA) and is undergoing irregular treatment with adalimumab. Antibiotic therapy and prednisone $1 \mathrm{mg} / \mathrm{kg} /$ day were prescribed to control vasculitis. On physical examination extensive necrotic area in L hallux and onset of necrosis in L second toe (Fig. 1). Left third and fourth toes were pale and cold without capillary filling. Foot radiograph shows severe erosions of the metatarsophalangeal joints, with almost no joint space left and the original bone outlines partially presented, showing advanced RA (Fig. 2 and 3 ). In the hands, there is a decrease in interphalangeal joint space and marginal erosion located in the distal metacarpals and proximal phalanges (Fig. 4). Laboratory tests:

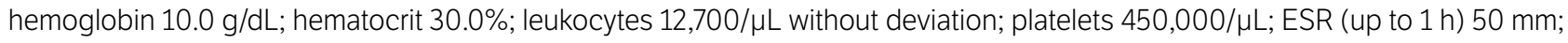
CRP100 mg/L; GOT23U/L; GPT24U/L; creatinine1.1 mg/dL; normal UPC; RF positive (1024IU/mL); Anti-CCP antibody positive(204IU/mL). Laboratory tests of the patient showed that RF was elevated (73 times the upper limit), as well as leukocytes (1.27 times), ESR (2.5 times) and CRP (33.3 times). In addition, the patient was anemic $(\mathrm{Hgb}<12 \mathrm{~g} / \mathrm{dL})$ with common extra-articular manifestation.

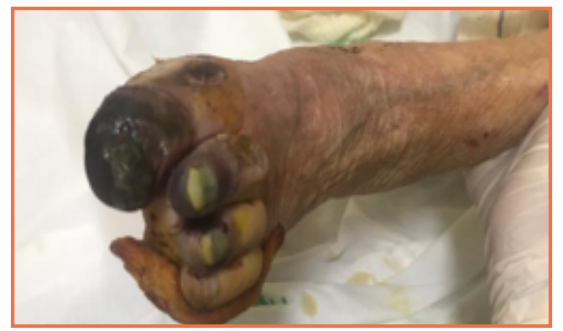

Figure 1. Presence of necrosis in hallux and second toes.

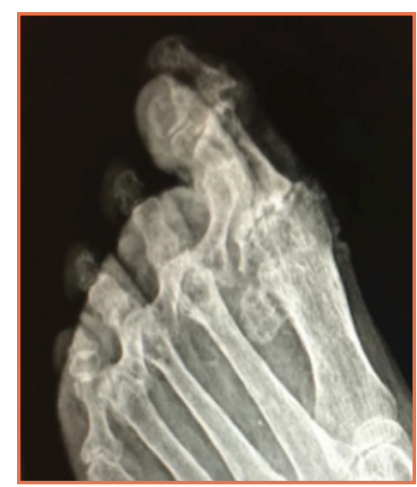

Figure 2. Radiolucent and deforming erosions on left first metatarsal head and proximal interphalangeal, showing osteonecrosis. 


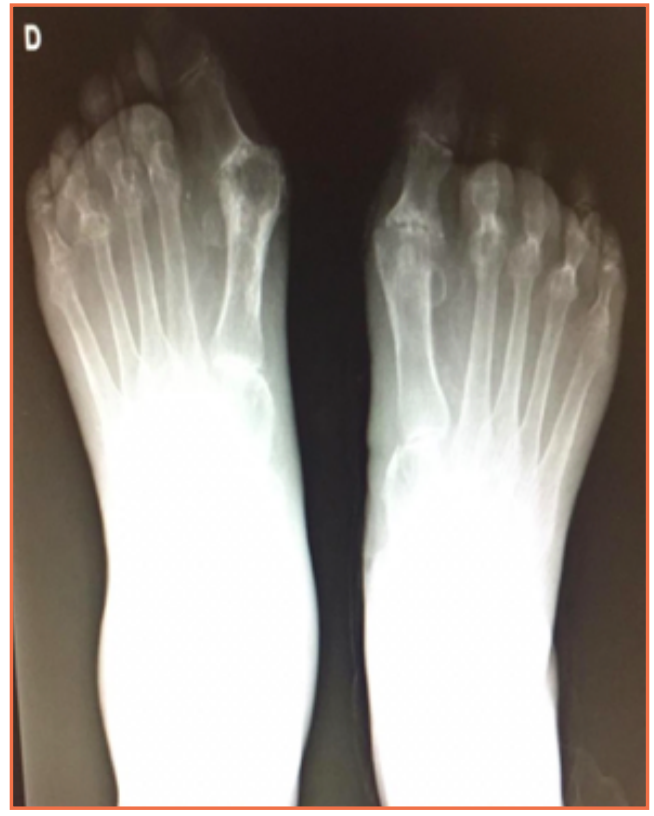

Figure 3. Reduction of joint space and erosion of metatarsophalangeal joints.

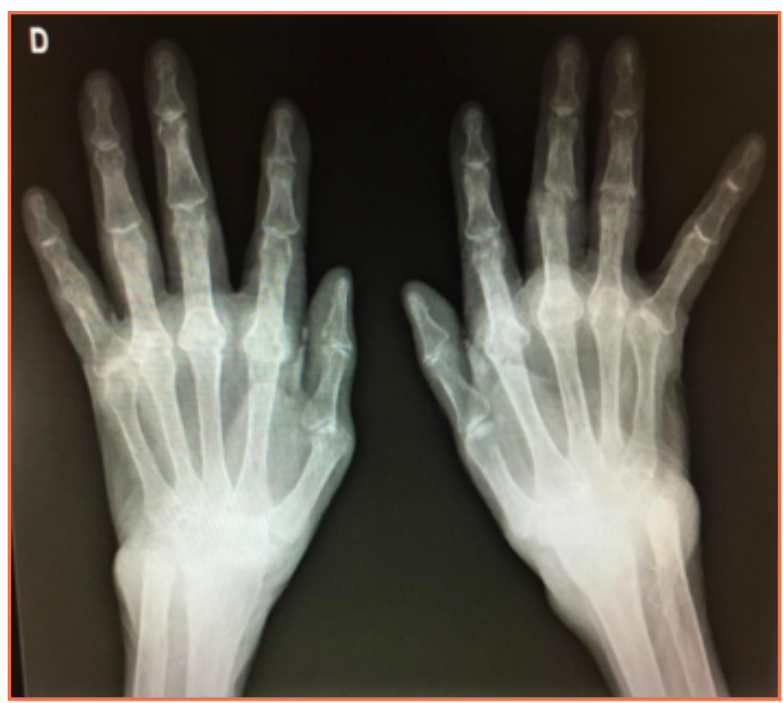

Figure 4. Erosion and decrease of metacarpophalangeal and interphalangeal joint space.

\section{CONCLUSION}

Although rare, it is important to keep an eye on the development of rheumatoid vasculitis in the clinical course of rheumatoid arthritis, since it has significant morbidity, justifying its recognition and early treatment. 